\title{
Melanie Werren (2019) Würde und Demenz. Grundlegung einer Pflegeethik
}

\author{
Nomos, Baden-Baden, 223 Seiten, 46,00 €, ISBN 978-8487-5546-2
}

\author{
Kathrin Kürsten
}

Angenommen: 30. März 2021 / Online publiziert: 20. April 2021

(C) Der/die Autor(en) 2021

Beim vorgestellten Werk handelt es sich um die Promotionsschrift (223 Seiten) der Autorin, in der zunächst die Grundsatzfrage gestellt wird: Verliert ein Mensch, der an Demenz erkrankt, seine Würde? In der inhaltlichen Auseinandersetzung wird sehr schnell deutlich, dass eine Antwort nicht en passant zu finden ist, denn es tut sich eine weitere, noch weitaus grundsätzlichere Frage auf: Was ist Würde?

Werren selbst ist evangelische Ethikerin und Pflegefachfrau. Als Praktikerin mahnt sie bereits im ersten Kapitel - der Einleitung - an, dass gerade die Pflegenden, die den meisten zwischenmenschlichen Kontakt mit an Demenz erkrankten Menschen haben, im öffentlichen Diskurs am wenigsten wahrgenommen werden. So ist es Werrens Ziel ,eine pflegeethische Grundlegung der Würdekategorie [...] zu entwickeln“ (S. 21). Dazu wählt sie ein interdisziplinäres Vorgehen und verknüpft Aspekte, insbesondere aus Pflege, Ethik und Philosophie.

Im darauffolgenden zweiten Kapitel „Problemzusammenhänge“ setzt sich Werren umgehend mit der grundlegenden Frage auseinander, was Würde bedeutet und welche Attribute einem Menschen Würde verleihen können. Dazu wird den Lesenden ein Einblick in den Sprachgebrauch des Würdebegriffs gewährt, womit die Autorin verdeutlicht, dass die Bedeutung des Wortes „Würde“ in seiner alltäglichen Verwendung sehr vage bleibt. Das Kapitel wird durch die Konkretisierung von Würde abgeschlossen. Würde wird im Zusammenhang mit Menschenrechten, der Autonomie des Einzelnen (hier wird das Problem eines informed consent im Zusammenhang mit einer dementiellen Erkrankung besonders hervorgehoben) sowie mit einer ,guten“ Umsetzung der praktischen Pflege gebracht.

K. Kürsten, M.A. ( $\square)$

Katholische Hochschule NRW, Köln, Deutschland

E-Mail: k.kuersten@katho-nrw.de

Philosophisch-Theologische Hochschule Vallendar, Vallendar, Deutschland 
Im Folgenden erfolgt eine explizite Auseinandersetzung mit unterschiedlichen Begründungsstrategien von Würde im Zusammenhang mit Demenz. Hierbei unterscheidet Werren in Rückbezug auf das vorherige Kapitel grundlegend zwischen zwei Konzeptionen von Würde, die sie kleinschrittig herausarbeitet:

1. unbedingte Würde: Würde eines Menschen begründet sich aus seinem Menschsein selbst.

2. bedingte Würde: Würde wird durch erworbene Attribute definiert.

Zunächst wird die unbedingte Würde sowohl mittels einer Vielzahl von Bibelstellen und evangelisch-theologischer Begründungen als auch am Würdebegriff Kants beleuchtet. Der unbedingten Würde wird allerdings attestiert, dass sie sehr abstrakt bleibt und nur wenige Anhaltspunkte für die praktische Umsetzung bietet.

Im zweiten Teil des dritten Kapitels setzt sich die Autorin mit der bedingten Würde auseinander und unterscheidet dabei zwischen zwei Begründungsstrategien von Würde: Leistung und Anerkennung. Diese beiden Aspekte legt Werren ausführlich dar und nimmt dabei Bezug zu vielerlei weiteren Perspektiven wie beispielsweise erneut dem Würdebegriff im biblischen Kontext oder bei Immanuel Kant.

Aufgrund der Tatsache, dass Menschen mit Demenz unweigerlich Gefahr laufen, ihre bedingte Würde zu verlieren, beschäftigt sie sich zusätzlich mit weiteren wissenschaftlichen Ansätzen, die beschreiben, wie dieser „Würdeverlust“ verhindert werden kann (so z. B. Martha Nussbaums Ausführungen zu „Human Dignity“). Es wird deutlich, dass für die Autorin die Konzepte der bedingten Würde - im Gegensatz zur o.g. Abstraktheit der unbedingten Würde - für die praktische Anwendung bedeutsam sind.

Im vierten Kapitel führt Werren nun alle bisherigen Gedankengänge zusammen und differenziert zwischen menschenrechtlichen Prinzipien und Kriterien der Anerkennung sowie der Leistung. Diese drei abgegrenzten Themenbereiche betrachtet sie jeweils aus individualethischer (Regulierung des menschlichen Miteinanders) und sozialethischer (Institutionen, Gesetze etc.) Perspektive. Aus dieser dezidierten Auseinandersetzung resultieren „Professionsethische Konsequenzen“, die anhand von sechs Spannungsfeldern - die im praktischen Alltag relevant werden können - beschrieben werden. So beispielsweise das Spannungsfeld zwischen der Forderung nach Selbstständigkeit und Teilhabe einerseits, jedoch andererseits ebenso die Anerkennung der Verletzlichkeit menschlichen Lebens und schlussendlich der Sterblichkeit.

Das fünfte Kapitel bildet einen recht kurzen Abschluss, indem Werren zunächst den in der Alltagssprache eher schwammigen Würdebegriff für die Pflege zusammenfassend präzisiert, indem sie gerade die oben genannten bedingten Würdekategorien erneut hervorhebt, die für eine gelungene Praxis in ein entsprechendes Interaktionsverhalten zwischen Pflegenden und Menschen mit Demenz münden müssen. Anschließend veranschaulicht die Autorin die praktische Bedeutung ihrer Erkenntnis und entwickelt zwei Tabellen, die die Ergebnisse ihrer Auseinandersetzung prägnant visualisieren und für Pflegende durchaus einen gehaltvollen Praxisbezug bieten.

Fazit: Der Autorin glückt eine anschauliche Darstellung des Würdebegriffes durch die Unterteilung in bedingte und unbedingte Würde. Die Bedeutung der Letzteren für ein würdevolles Leben mit einer dementiellen Veränderung kann als Grundlegung 
einer Pflegeethik verstanden werden. So offenbart Werren, dass die allumfassende Definition der unbedingten Würde für eine praktische Umsetzung nicht ausreicht. Denn was bedeutet diese für das alltagspraktische Handeln von Pflegenden? Zunächst mag es befremdlich erscheinen, dass gerade die bedingte Würde (Leistung und Anerkennung) zur Beantwortung dieser Frage unabdingbar ist. Denn eben jene, im Laufe des Lebens erworbenen Elemente, gehen bei einer Demenz verloren. Allerdings ist es gerade der Ausgleich dieses ,Verlustes“ durch die entsprechende Interaktion, insbesondere mit Pflegenden, der die Würde für Menschen mit Demenz aufrechterhalten kann. Auf diesem Weg gelingt es der Autorin „Kriterien zur Konkretion“"(S. 176) zu entwickeln, die einen wertvollen, weil auch praxisnahen Beitrag leisten. Diese Konkretionen sollten für eine gelingende Konzeptentwicklung in der Pflegepraxis bzw. im Qualitätsmanagement genutzt werden. In seiner Gesamtheit ist die Dissertationsschrift allerdings für ein wissenschaftliches Fachpublikum empfehlenswert.

Funding Open Access funding enabled and organized by Projekt DEAL.

Open Access Dieser Artikel wird unter der Creative Commons Namensnennung 4.0 International Lizenz veröffentlicht, welche die Nutzung, Vervielfältigung, Bearbeitung, Verbreitung und Wiedergabe in jeglichem Medium und Format erlaubt, sofern Sie den/die ursprünglichen Autor(en) und die Quelle ordnungsgemäß nennen, einen Link zur Creative Commons Lizenz beifügen und angeben, ob Änderungen vorgenommen wurden.

Die in diesem Artikel enthaltenen Bilder und sonstiges Drittmaterial unterliegen ebenfalls der genannten Creative Commons Lizenz, sofern sich aus der Abbildungslegende nichts anderes ergibt. Sofern das betreffende Material nicht unter der genannten Creative Commons Lizenz steht und die betreffende Handlung nicht nach gesetzlichen Vorschriften erlaubt ist, ist für die oben aufgeführten Weiterverwendungen des Materials die Einwilligung des jeweiligen Rechteinhabers einzuholen.

Weitere Details zur Lizenz entnehmen Sie bitte der Lizenzinformation auf http://creativecommons.org/ licenses/by/4.0/deed.de.

Interessenkonflikt K. Kürsten gibt an, dass kein Interessenkonflikt besteht. 